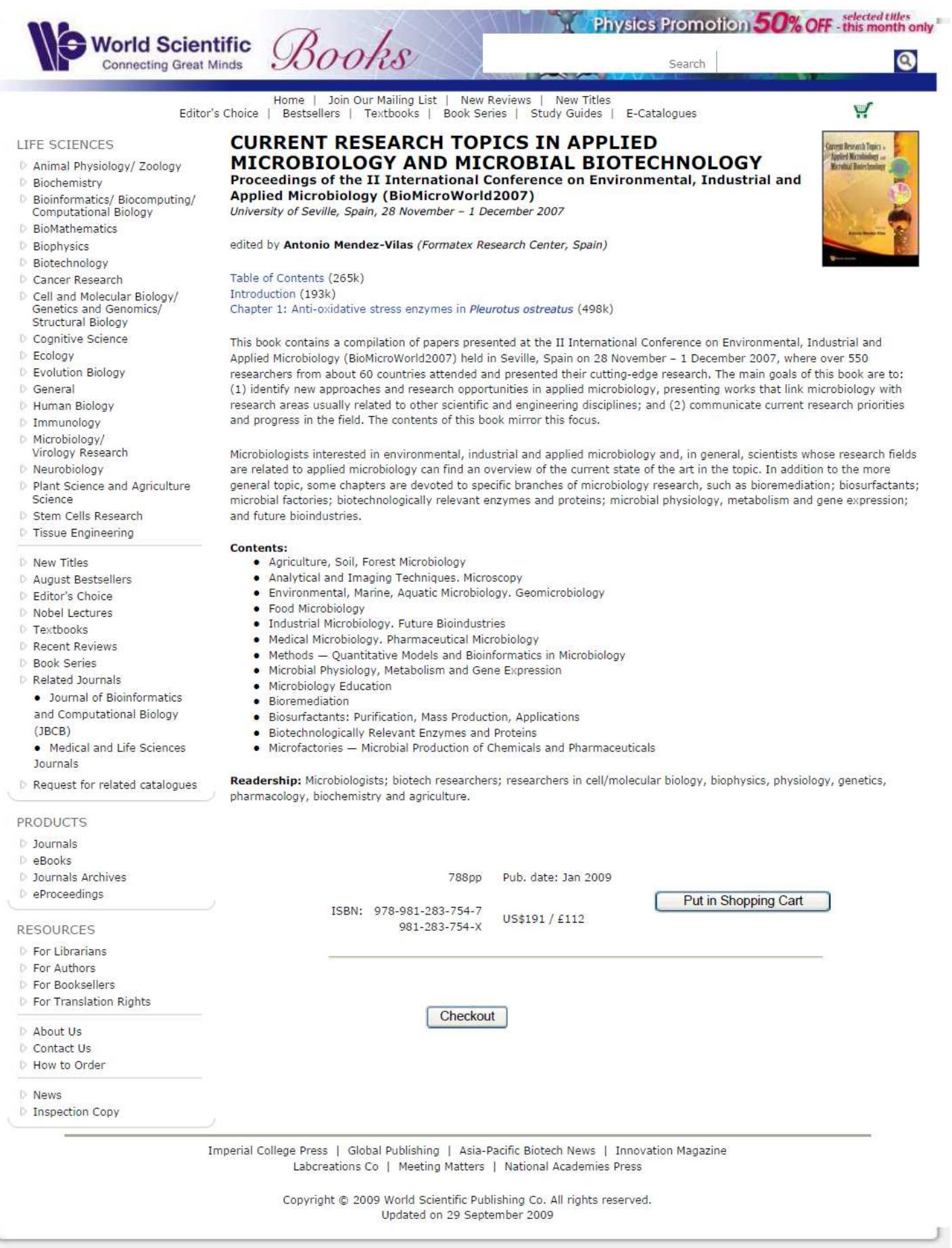




\title{
Yeast stress enzymes - application of microbiology and bioinformatics for initiate high school students in environmental studies
}

\author{
I. Alves-Pereira ${ }^{1,2}$ and R. Ferreira ${ }^{*}, 1,2$ \\ ${ }^{1}$ Departamento de Química, Universidade de Évora, Apartado 94, 7002-554 Évora, Portugal \\ ${ }^{2}$ Instituto de Ciências Agrárias Mediterrânicas (ICAM), Apartado 94, 7002-554 Évora, Portugal
}

The aim of this work was to prepare a summer course for high school students of Portugal which illustrate the importance of microbiology and bioinformatics applications in environmental studies, emphasizing that molecular mechanisms of response, repair and adaptation, endows the cell with essential plasticity to adjust to environmental events, by a process termed stress response. Five high school students with ages ranging from 15 to 17 years old are executes in our laboratory very simple experiments observing that vanadium presence in culture medium, switch on a yeast stress response. This course covers the genomic and functional characterization of CAT T from yeast by bioinformatics search, experimental detection and its response to the vanadium presence in culture medium. The obtained results, namely CAT T detection, its positive response to vanadium and structural and metabolic characterization of gene CTT 1 products reveal to the students the importance of yeast enzymatic detection as environmental response markers.

Keywords vanadium stress; catalase;, Saccharomyces cerevisiae

" Corresponding author: e-mail: raf@uevora.pt, Phone: +351 266745300 